www.nature.com/ja

\title{
Diversity of nonribosomal peptide synthetase and polyketide synthase genes in the genus Actinoplanes found in Mongolia
}

\author{
Jigjiddorj Enkh-Amgalan ${ }^{1}$, Hisayuki Komaki ${ }^{2}$, Damdinsuren Daram ${ }^{1}$, Katsuhiko Ando ${ }^{2}$ and Baljinova Tsetseg ${ }^{1}$
}

The Journal of Antibiotics (2012) 65, 103-108; doi:10.1038/ja.2011.115; published online 14 December 2011

Keywords: Actinoplanes; antimicrobial activity; nonribosomal peptide synthetase; polyketide synthase

\begin{abstract}
Mongolia has an undisturbed ecosystem with rich biodiversity, but only a few research groups have focused attention on the region for its actinomycetes diversity and their antimicrobial activities. ${ }^{1,2}$ The genus Actinoplanes is representative of rare actinomycetes and the reported source of more than 120 antibiotics. ${ }^{3}$ This study was designed to assess the individual abilities of taxonomically diverse Actinoplanes strains, isolated from Mongolian soil, to produce biologically active compounds. First, the antimicrobial activities of culture samples were examined, using a conventional assay that was facile and suitable for preliminary screening, to check the strains' abilities to produce antibiotics. Discrimination of distinct strains and identification of the producers of already reported compounds, called dereplication, were the next important steps for efficient screening and further assessing the isolates. In contrast to culture-dependent methods, genetic analysis is not influenced by culture conditions, and thus can address genes unexpressed under normal laboratory culture conditions. As nonribosomal peptides and polyketides represent two of the major antibiotic classes prevalent in the genus Actinoplanes, ${ }^{4-7}$ two biosynthetic gene types, nonribosomal peptide synthetase (NRPS) and polyketide synthase (PKS) genes, were analyzed to evaluate the isolates' potential for production of these types of compounds. ${ }^{8-10}$ In this study, the diversity of the NRPS and PKS genes in these isolates as well as their relationships in Actinoplanes taxonomy were investigated.

In July 2007, 39 soil samples were collected at three sampling sites in Tov, Uvs and Dornad Provinces, Mongolia, and actinomycetes were isolated from these samples. Genomic DNA was extracted using FastBreak Cell Lysis Reagent, SV Lysis Buffer and MagneSil RED (Promega Corp., Madison, WI, USA). Polymerase chain reaction (PCR) amplification of $16 \mathrm{~S}$ rDNA was performed as described by Tamura et al. ${ }^{11}$ Purified PCR products were directly sequenced, using an ABI Prism BigDye Terminator Cycle Sequencing Kit and a 3730xl
\end{abstract}

Genetic Analyzer (Applied Biosystems, CA, USA). For phylogenetic analysis, the $16 \mathrm{~S}$ rDNA sequences were aligned with genus Actinoplanes reference sequences using the CLUSTAL_X software and a phylogenetic tree constructed using the neighbor-joining method. ${ }^{12}$

Adenylation (A) domain regions in NRPS genes, ketosynthase (KS) domain regions in type-I PKS genes and KS $\alpha$ genes in type-II PKS genes were amplified using specific primer sets described by AyusoSacido et al. ${ }^{8}$ Schermer et al. ${ }^{13}$ and Metsa-Ketela et al., ${ }^{14}$ respectively. The PCR products were cloned, sequenced and searched by BLASTX on the NCBI website and phylogenetic trees were constructed. ${ }^{10,14}$ The substrate-specificity-conferring residues, comprising the aminoacid (aa)-binding pocket ${ }^{15}$ and the substrate aa of A domains in the NRPS genes were predicted using the PKS/NRPS Analysis Web-site (http://nrps.igs.umaryland.edu/nrps/).

The 398 actinomycete strains, isolated and analyzed as described above, were preserved at the Institute of Biology, Mongolian Academy of Sciences and National Institute of Technology and Evaluation (http://www.bio.nite.go.jp/nbdc/e/). According to their 16S rDNA sequences, these isolates were found to comprise 250 Streptomyces strains and 148 rare actinomycetes including 23 Actinoplanes strains. With the present focus on the genus Actinoplanes, all Actinoplanes isolates were selected for further analysis (Table 1). They shared 98-100\% identity with known Actinoplanes species according to a BLAST search of their 16S rDNA sequences and a phylogenetic analysis based on their $16 \mathrm{~S} \mathrm{rDNA}$ sequences revealed a taxonomically diverse collection (Figure 1a).

All 23 isolates were initially tested for antimicrobial activity using agar-plug assays. ${ }^{16}$ Thirteen strains (57\%) were active against Grampositive Bacillus subtilis and Staphylococcus aureus, two (9\%) of which were also active against Gram-negative Escherichia coli. Also, three strains (13\%) exhibited anti-yeast activities against Saccharomyces

${ }^{1}$ Institute of Biology, Mongolian Academy of Sciences (MAS), Ulaanbaatar, Mongolia and ${ }^{2}$ NITE Biological Resource Center (NBRC), National Institute of Technology and Evaluation (NITE), Chiba, Japan

Correspondence: Dr H Komaki, NITE Biological Resource Center (NBRC), National Institute of Technology and Evaluation (NITE), 2-5-8 Kazusakamatari, Kisarazu, Chiba 292-0818, Japan.

E-mail: komaki-hisayuki@nite.go.jp

Received 27 September 2011; revised 10 November 2011; accepted 14 November 2011; published online 14 December 2011 
cerevisiae and five strains (22\%) showed antifungal activities against Aspergillus awamori (Table 1). Collectively, 65\% of the isolates exhibited antimicrobial activity against one or more of the test organisms. Notably, two strains (MN07-A0344 and A0367) showed inhibitory effects against all test organisms, whereas nine strains (MN07-A0329, A0351, A0360, A0362, A0363, A0364, A0365, A0369 and A0373) were specifically active against Gram-positive bacteria. In addition, two strains (MN07-A0335 and A0359) showed similar antimicrobial spectra with anti-Gram-positive and antifungal activities, whereas the activity of MN07-A0330 and A0349 were specific for fungi and yeast, respectively. The antimicrobial spectra of some phylogenetically close strains (MN07-A0362, A0363, A0364 and A0365) were identical, but it was also observed that phylogenetically distant and diverse strains (MN07-A0351, A0369, A0373, A0329 and A0360) also showed similar spectra. Meanwhile, the antimicrobial spectra of MN07-A0325, A0329 and A0344 differed considerably from each other although the three strains were phylogenetically identical.
Next, PCR assays were used to detect the presence in the isolates of NRPS genes and two kinds of PKS genes, type-I PKSs, known as modular enzymes, and type-II PKSs, mostly responsible for the synthesis of aromatic polyketides. Distinct DNA amplifications possessing the expected sizes for NRPS and type-I and -II PKS genes were observed in 17, 17 and 14 of the strains, respectively. The detected genes were further investigated by cloning and sequencing of their PCR products.

Among 68 clones sequenced from the 17 NRPS-positive strains, 39 different sequences were obtained (Supplementary Table S1). A BLASTX search showed their sequence identities, relative to already published NRPS genes, ranged from $49 \%$ (58\% similarity) to $83 \%$ ( $88 \%$ similarity). The most BLAST hits were NRPS genes found by genome-sequencing projects on actinomycetes, such as Streptomyces and Pseudonocardia, and whose metabolites have not been identified. To determine the diversity of the 39 sequences, the deduced aa sequences were compared using a phylogenetic tree (Figure 1b). Except for two clone pairs, MN07-A0344-\#7/MN07-A0329-\#1 and

Table 1 Isolated Actinoplanes strains and their antimicrobial activities

\begin{tabular}{|c|c|c|c|c|c|c|}
\hline \multirow[b]{2}{*}{ Strain } & \multirow[b]{2}{*}{ Closest species based on $16 S$ rDNA sequence (similarity) } & \multicolumn{5}{|c|}{ Inhibition zone $(\mathrm{mm})$ against } \\
\hline & & Bs & Sa & Ec & Sc & Aa \\
\hline MN07-A0325 & A. nipponensis (99\%) & - & - & - & - & - \\
\hline MN07-A0326 & A. nipponensis (99\%) & - & - & - & - & - \\
\hline MN07-A0329 & A. nipponensis (99\%) & 14 & 13 & - & - & - \\
\hline MN07-A0330 & A. friuliensis $(98 \%)$ & - & - & - & - & 10 \\
\hline MN07-A0335 & A. auranticolor (99\%) & 11 & 10 & - & - & 16 \\
\hline MN07-A0344 & A. nipponensis (99\%) & 27 & 26 & 17 & 12 & 21 \\
\hline MN07-A0345 & A. nipponensis (99\%) & - & - & - & - & - \\
\hline MN07-A0347 & A. rectilineatus (98\%) & - & - & - & - & - \\
\hline MN07-A0348 & A. utahensis (98\%) & - & - & - & - & - \\
\hline MN07-A0349 & A. nipponensis (99\%) & - & - & - & 10 & - \\
\hline MN07-A0351 & A. palleronii (99\%) & 12 & 12 & - & - & - \\
\hline MN07-A0359 & A. violaceus (99\%) & 15 & 16 & - & - & 12 \\
\hline MN07-A0360 & A. digitatis (98\%) & 10 & 11 & - & - & - \\
\hline MN07-A0361 & A. arizonaensis (99\%) & - & - & - & - & - \\
\hline MN07-A0362 & A. cyaneus $(99 \%)$ & 10 & 11 & - & - & - \\
\hline MN07-A0363 & A. garbadinensis (99\%) & 11 & 11 & - & - & - \\
\hline MN07-A0364 & A. cyaneus $(99 \%)$ & 11 & 10 & - & - & - \\
\hline MN07-A0365 & A. garbadinensis (99\%) & 12 & 10 & - & - & - \\
\hline MN07-A0367 & A. humidus (98\%) & 19 & 20 & 12 & $\operatorname{Tr}$ & 12 \\
\hline MN07-A0368 & A. toevensis $(100 \%)$ & - & - & - & - & - \\
\hline MN07-A0369 & A. deccanensis (98\%) & 23 & 20 & - & - & - \\
\hline MN07-A0371 & A. tereljensis (100\%) & - & - & - & - & - \\
\hline MN07-A0373 & A. auranticolor (98\%) & 16 & 13 & - & - & - \\
\hline
\end{tabular}

Abbreviations: Aa, Aspergillus awamori; Bs, Bacillus subtilis; Ec, Escherichia coli; Sa, Staphylococcus aureus; Sc, Saccharomyces cerevisiae; Tr, trace (incomplete inhibition). -No inhibition observed.

Figure 1 Phylogenetic trees based on (a) 16S rDNA sequences, (b) A domain sequences ( $\sim 230$ aa) in NRPS genes, (c) KS domain sequences ( $\sim 210$ aa) in type-I PKS genes and (d) KS $\alpha$ gene sequences ( $570 \mathrm{bp}$ ) in type-II PKS genes. Mongolian isolates bold-faced in (a); strain (MNO7-) and clone no. (Supplementary Table 1) in (b, c and d); Pst and Tei/Tcp, already reported from genus Actinoplanes, involved in friulimicin synthesis and teicoplanin synthesis, respectively, ${ }^{6,7}$ added as references; specificity-conferring 8 aa residues ${ }^{15}$ and predicted substrate aa in brackets, some aa residues shown as $\mathrm{X}$ and some substrate aa could not be determined in the PKS/NRPS Analysis Web-site in (b); identity to closest PKS in BLASTX search in brackets in (c); reference genes with identified metabolites shown with gene name; if metabolites unidentified, strains with $\mathrm{KS} \alpha$ genes in (d); bootstrap values $>50 \%$ from 1000 replicates shown at nodes. 
Actinoplanes regularis DSM 43151 ${ }^{\top}$ (AB037011)

Actinoplanes sp. MN07-A0351

${ }_{3}\left[-\right.$ Actinoplanes palleronii DSM $43940^{\top}$ (AJ277573)

Actinoplanes digitatis DSM 43149 (AJ277567)

Actinoplanes missouriensis DSM 43046 ${ }^{\top}$ (AJ277572)

Actinoplanes rectilineatus DSM 43808 ${ }^{\top}$ (AB037010)

Actinoplanes sp. MN07-A0347

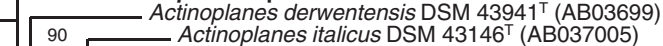

Actinoplanes couchii GW81761 (AM400230)

98 Actinoplanes sp. MN07-A0361

$62 \quad$ - Actinoplanes xinjiangensis 03-8772 ${ }^{\top}$ (EU531457)

Actinoplanes sichuanensis 03-723 $3^{\top}$ (EU531458)

90 Actinoplanes campanulatus DSM 43148 (AB036995)

$55 \quad 99 \quad$ Actinoplanes lobatus DSM 43150 (AB037006)

- Actinoplanes auranticolor DSM 43031 $1^{\top}$ (U58527)

60 Actinoplanes utahensis DSM 43147 (X80823)

99 Actinoplanes sp. MN07-A0364

99 Actinoplanes sp. MN07-A0365

$100 \square$ Actinoplanes liguriae DSM 43865 ${ }^{\top}$ (AJ865471)

- Actinoplanes cyaneus DSM 46137' (AB036997)

${ }_{54}$ - Actinoplanes sp. MN07-A0362

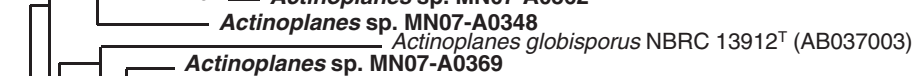

92 - Actinoplanes deccanensis DSM 43806
T

Actinoplanes sp. MN07-A0330

Actinoplanes sp. MN07-A0367

$100 \quad$ Actinoplanes humidus DSM 43938 ${ }^{\top}$ (AB037004)

Actinoplanes consettensis DSM 43942 ${ }^{\top}$ (AB036996)
Actinoplanes sp. MN07-A0373

Actinoplanes sp. MN07-A0335

Actinoplanes sp. MN07-A0359

99 Actinoplanes sp. MN07-A0345

Actinoplanes sp. MN07-A0326

91 Actinoplanes sp. MN07-A0349

Actinoplanes sp. MN07-A0344
Actinoplanes sp. MN07-A0329

Actinoplanes sp. MN07-A0325

Actinoplanes sp. MN07-A0360

Actinoplanes brasiliensis DSM $43805^{\top}$ (X93185)

Actinoplanes durhamensis JCM $7625^{\top}$ (AB048215)

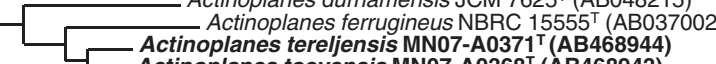

91 Actinoplanes toevensis MN07-A0368 ${ }^{\top}$ (AB468943)

Micromonospora chalcea DSM $43026^{\top}$ (X92594)

b

NRPS

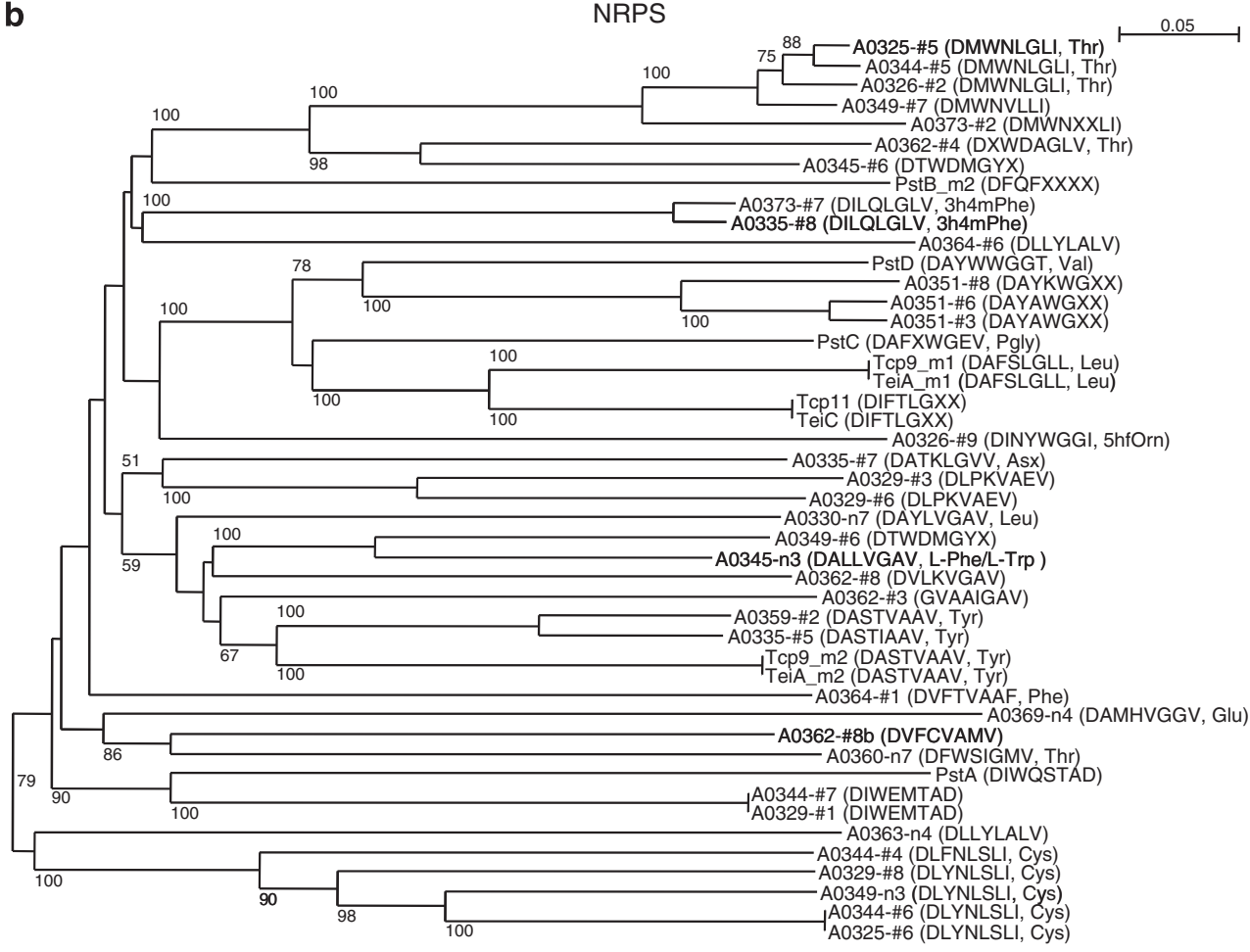




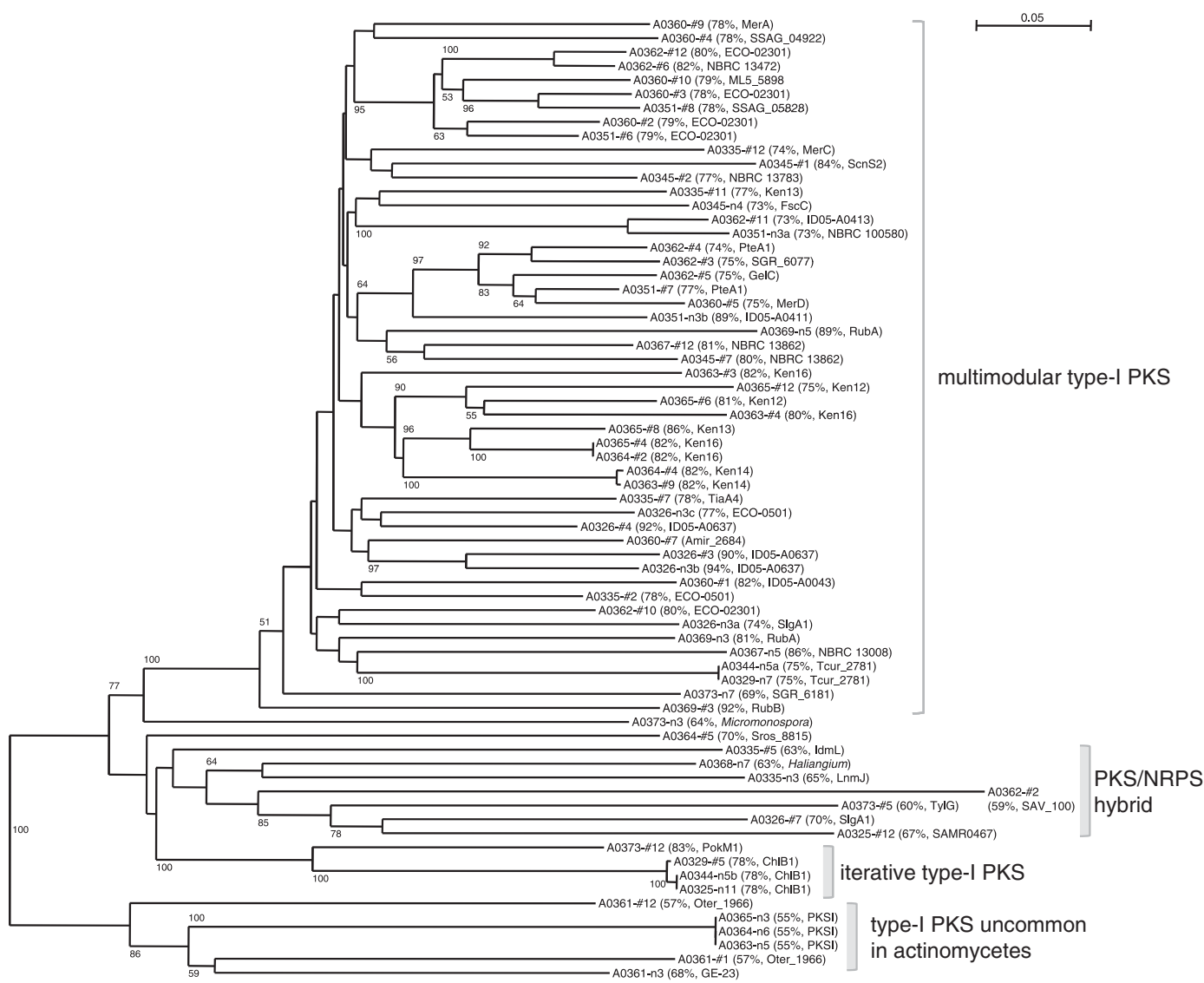

d

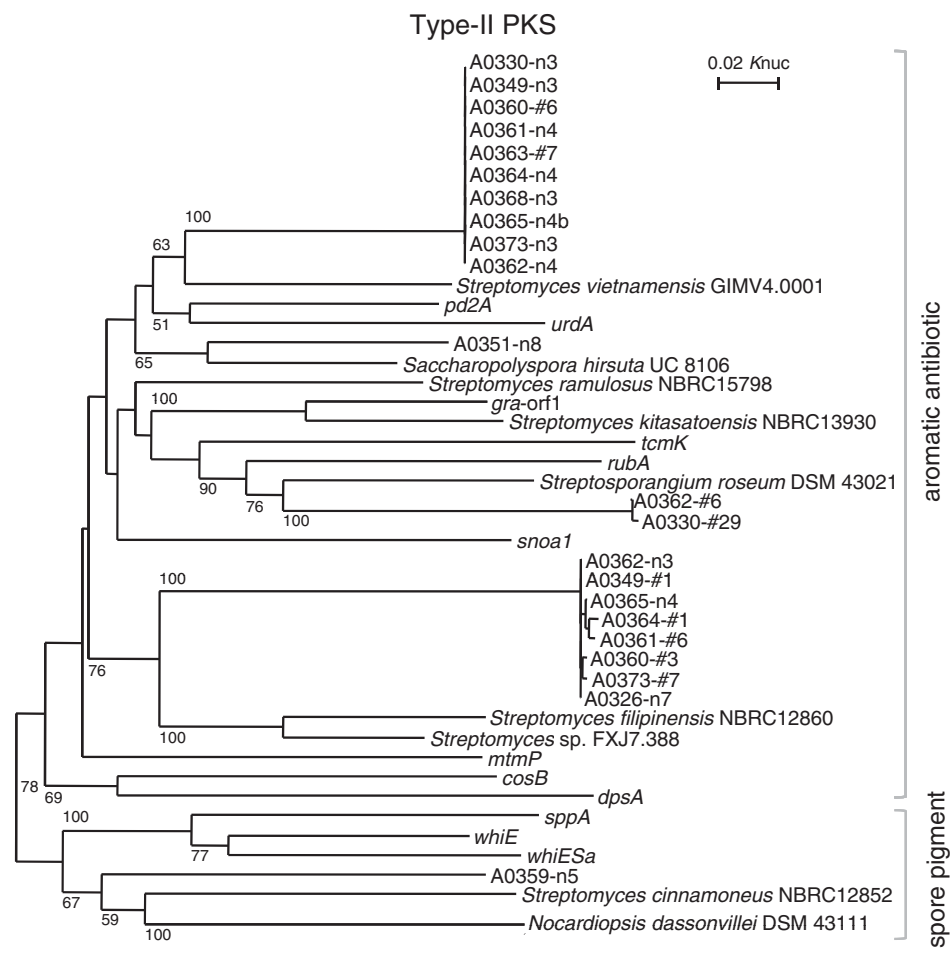

Figure 1 Continued. 
MN07-A0325-\#6/MN07-A0344-\#6, no identical-sequence pairs were observed, suggesting NRPS gene-sequence diversity among the 17 strains. Although teicoplanins and friulimicins are known as representative nonribosomal peptide metabolites in the genus Actinoplanes, ${ }^{6,7}$ no tcp/tei- or pst-like genes corresponding to these compounds were detected in these strains. The specificity-conferring 8 aa residues of the A domains and the predicted aa substrates are also shown in Figure 1b. The substrates were closely related with the phylogenetic position of each gene sequence. Threonine (Thr)- and cysteine (Cys)-recognizing NRPS sequences were often observed, and the isolates also possessed other NRPS sequences that recognized various aa. Interestingly, phylogenetically close strains were found to often share similar NRPS sequences. For instance, similar sequences of MN07-A0355-\#8 and A0373-\#7 (Supplementary Table S1), which recognize 3-hydroxy-4-methoxyphenylalanine (Figures $1 \mathrm{~b}$ ) as the substrate, were observed in the phylogenetically close MN07-A0335 and A0373, respectively. Thr- and Cys-recognizing sequences were also distributed among phylogenetically close strains, such as MN07A0325, A0344 and A0349. Indeed, the substrates of many NRPS sequences could not be predicted because their specificity-conferring 8 aa residues showed no correspondence in the related database; thus, their involvement in the synthesis of peptide compounds, including unusual aa, could be expected.

Analyses of 159 independent clones from 17 type-I PKS-positive strains yielded 70 different DNA sequences (Supplementary Table S1). Their sequence identities relative to already published type-I PKS genes ranged from $55 \%$ ( $64 \%$ similarity) to $94 \%$ ( $96 \%$ similarity). Three of six clones derived from MN07-A0329 showed over $90 \%$ identity with PKS genes of an Actinoplanes strain. ${ }^{17}$ MN07-A0369 possessed $r u b$-like genes probably involved in rubradirin synthesis. ${ }^{18}$ Type-I PKS-coding DNA fragments, showing $74-86 \%$ identity to kendomycin synthetic genes (ken), ${ }^{19}$ were observed in MN07-A0335, A0363, A0364 and A0365. Except for these sequences, the novelty of many other sequences was comparatively high. Next, the sequence diversity of the isolates' type-I PKSs was checked by phylogenetic analysis (Figure 1c). Although identical or very similar sequences were infrequently observed among phylogenetically identical strains, such as MN07-A0363 and A0364, A0365 and A0325, or A0329 and A0344, most detected sequences were diverse. Phylogenetic analysis indicated that typical multimodular type-I PKS genes constituted the majority of these genes; however, unusual PKSs, likely to be PKS/ NRPS hybrids or iterative types, are also often present. Furthermore, the sequences of A0365-n3/A0364-n6/A363-n5, A0361-\#1 and A0361-n3 were novel and considered uncommon in actinomycetes as almost all 50 BLAST hits were type-I PKSs in non-actinomycetes (data not shown), such as Rhodobacteraceae bacterium and Opitutus. This is the first report suggesting the existence of unusual type-I PKS genes in the genus Actinoplanes, there being no registration of similar unusual type-I PKS genes derived from Actinoplanes strains in GenBank/ EMBL/DDBJ.

Sequencing of 75 clones derived from 14 type-II PKS positive strains revealed 23 independent DNA sequences (Supplementary Table S1). Among them, eight strains showed amplification of multiple sequences, with MN07-A0362 yielding three kinds of sequences and MN07-A0330, A0349, A0360, A0361, A0364, A0365 and A0373 each yielding two kinds of sequences. On the other hand, only a single sequence was obtained from the remaining six strains. As type-II PKSs do not have a modular organization, a gene cluster usually encodes only a single KS $\alpha$. Therefore, MN07-A0362, the seven, and the six strains grouped above were considered to possess at least three, two and one type-II PKS gene clusters, respectively. Their sequence identities relative to already published type-II PKS genes ranged from $80 \%$ ( $88 \%$ similarity) to $86 \%$ ( $93 \%$ similarity). Most BLAST hits were KS $\alpha$ of Streptomyces sp. FXJ7.388 (accession, ADW09320) or Streptomyces vietnamensis (ACQ84440) and phylogenetic analysis revealed that the two types of sequences closest to them were shared by taxonomically diverse strains, such as MN07-A0361, A0364, A0373 and A0360, although four clones, A0351-n8, A0362-\#6, A330-\#29 and A0359-n5, were not included (Figure 1d). This suggested that their type-II PKS gene diversity was low in contrast to their NRPS and type-I PKS genes. Whereas the type-II PKS pathway is known to be involved in aromatic antibiotic synthesis, it is also involved in spore pigment synthesis; the enzymes for antibiotic biosynthesis can be phylogenetically separated from those involved in spore pigment formation. ${ }^{14}$ Except for a sequence named A0359-n5, all sequences amplified from these isolates were likely involved in antibiotic synthesis (Figure 1d).

Among the eight strains that showed no antimicrobial activity (Table 1), three strains were negative in all PCR assays. Hence, the biosynthetic potential of these three strains appeared poor. On the other hand, NRPS and/or PKS genes were amplified from five of the eight strains, suggesting that special conditions might be required to express these cryptic genes and/or that the metabolites synthesized by these pathways might not have antimicrobial activity but may possess other bioactivities. Except for these five strains, the existence of NRPS and PKS genes was well correlated with antibiotic production. As $74 \%, 74 \%$ and $61 \%$ of the isolates possessed NRPS, type-I and type-II PKS genes, respectively, they appear to be a useful resource with significant genetic potential.

Recent genome projects have revealed that some actinomycete families, such as Streptomyces, Kitasatospora, Salinispora, Micromonospora and Saccharopolyspora, regularly possess several NRPS and PKS pathways; ${ }^{20}$ however, the whole genome sequence of the genus Actinoplanes has not been published to date (www.bio.nite.go.jp/ ngac/e/project-e.html), and reports regarding NRPS and PKS gene analysis are also limited, resulting in the small number of published NRPS and PKS genes in Actinoplanes. ${ }^{6,7,21}$ To our knowledge, this is the first report focused on the genus Actinoplanes and the results obtained here strongly supported the conclusion that the secondary metabolite gene variety observed here in the genus Actinoplanes was comparable to those of the genome-sequenced genus. This is because most Actinoplanes strains analyzed here contained novel and diverse NRPS or PKS genes or both.

This study revealed significant genetic diversity and high antibiotic biosynthetic potential in Actinoplanes strains isolated from Mongolian soil samples. Structural determination of the antibiotics they produce is the important next step in further studies. Purification and structural elucidation of the metabolites was not performed here; these strains are available for researchers upon request under the Convention on Biological Diversity principle. In conclusion, the Actinoplanes strains investigated here appeared to have great potential as a valuable source for the future discovery of novel biologically active agents.

The accession numbers of the DNA sequences determined here are AB672891-AB673022.

\section{ACKNOWLEDGEMENTS}

This work was carried out under the Joint Research Project between the Department of Biotechnology, National Institute of Technology and Evaluation and the Institute of Biology, Mongolian Academy of Sciences. We are grateful to Ms Rieko Kasaishi-Suzuki for DNA sequencing. 
1 Norovsuren, Z., Oborotov, G. V., Zenova, G. M., Aliev, R. A. \& Zviagintsev, D. G. [Haloalkaliphilic actinomycetes in soils of Mongolian desert steppes]. Izv. Akad. Nauk. Ser. Biol. 501-507 (2007).

2 Rachev, R. et al. Orange pigments of Streptomyces sp. C-72. I. Purification and antimicrobial activity. Acta. Microbiol. Bulg. 21, 57-61 (1987).

3 Lazzarini, A., Cavaletti, L., Toppo, G. \& Marinelli, F. Rare genera of actinomycetes as potential producers of new antibiotics. Antonie Leeuwenhoek 78, 399-405 (2000).

4 Cavalleri, B., Arnone, A., Di Modugno, E., Nasini, G. \& Goldstein, B. P. Structure and biological activity of lipiarmycin B. J. Antibiot. 41, 308-315 (1988).

5 Coronelli, C., Pagani, H., Bardone, M. R. \& Lancini, G. C. Purpuromycin, a new antibiotic isolated from Actinoplanes ianthinogenes N. sp. J. Antibiot. 27, 161-168 (1974).

6 Muller, C et al. Sequencing and analysis of the biosynthetic gene cluster of the lipopeptide antibiotic Friulimicin in Actinoplanes friuliensis. Antimicrob. Agents. Chemother. 51, 1028-1037 (2007)

7 Sosio, M. et al. Organization of the teicoplanin gene cluster in Actinoplanes teichomyceticus. Microbiology 150, 95-102 (2004).

8 Ayuso, A. et al. A novel actinomycete strain de-replication approach based on the diversity of polyketide synthase and nonribosomal peptide synthetase biosynthetic pathways. Appl. Microbiol. Biotechnol. 67, 795-806 (2005).

9 Khan, S. T. et al. Streptomyces associated with a marine sponge Haliclona sp.; biosynthetic genes for secondary metabolites and products. Environ. Microbiol. 13, 391-403 (2011).

10 Komaki, H. et al. Discovery of a pimaricin analog JBIR-13, from Streptomyces bicolor NBRC 12746 as predicted by sequence analysis of type I polyketide synthase gene. Appl. Microbiol. Biotechnol. 83, 127-133 (2009).

11 Tamura, T. \& Hatano, K. Phylogenetic analysis of the genus Actinoplanes and transfer of Actinoplanes minutisporangius Ruan et al. 1986 and 'Actinoplanes aurantiacus' to
Cryptosporangium minutisporangium comb. nov. and Cryptosporangium aurantiacum sp. nov. Int. J. Syst. Evol. Microbiol. 51, 2119-2125 (2001).

12 Saitou, N. \& Nei, M. The neighbor-joining method: a new method for reconstructing phylogenetic trees. Mol. Biol. Evol. 4, 406-425 (1987).

13 Schirmer, A. et al. Metagenomic analysis reveals diverse polyketide synthase gene clusters in microorganisms associated with the marine sponge Discodermia dissoluta. Appl. Environ. Microbiol. 71, 4840-4849 (2005).

14 Metsa-Ketela, M. et al. Molecular evolution of aromatic polyketides and comparative sequence analysis of polyketide ketosynthase and 16S ribosomal DNA genes from various streptomyces species. Appl. Environ. Microbiol. 68, 4472-4479 (2002).

15 Stachelhaus, T., Mootz, H. D. \& Marahiel, M. A. The specificity-conferring code of adenylation domains in nonribosomal peptide synthetases. Chem. Biol. 6, 493-505 (1999).

16 Ichikawa, T., Date, M., Ishikura, T. \& Ozaki, A. Improvement of kasugamycin-producing strain by the agar piece method and the prototroph method. Folia. Microbiol. 16, 218-224 (1971).

17 Komaki, H. et al. Diversity of type-l polyketide synthase genes in actinomycetes isolated from Indonesia in 2005. 14th International Symp. on the Biology of Actinomycetes, No. 047, 215, Newcastle (2007).

18 Sohng, J. K., Oh, T. J., Lee, J. J. \& Kim, C. G. Identification of a gene cluster of biosynthetic genes of rubradirin substructures in S. achromogenes var. rubradiris NRRL3061. Mol. Cells 7, 674-681 (1997).

19 Wenzel, S. C., Bode, H. B., Kochems, I. \& Muller, R. A type I/type III polyketide synthase hybrid biosynthetic pathway for the structurally unique ansa compound kendomycin. Chembiochem 9, 2711-2721 (2008).

20 Nett, M., Ikeda, H. \& Moore, B. S. Genomic basis for natural product biosynthetic diversity in the actinomycetes. Nat. Prod. Rep. 26, 1362-1384 (2009).

21 Nishida, H. et al. Generation of novel rapamycin structures by microbial manipulations. J. Antibiot. 48, 657-666 (1995).

Supplementary Information accompanies the paper on The Journal of Antibiotics website (http://www.nature.com/ja) 